Case Report

\title{
Additional M uscle Belly and Abberant Muscle Fibers over the Extensor Retinaculam of Wrist
}

\author{
Divia Paul A. ${ }^{1} \&$ Manisha Rajanand Gaikwad ${ }^{2}$ \\ ${ }^{1}$ Junior Research Fellow, Department of Anatomy, Yenepoya M edical College, M angalore, Karnataka, \\ ${ }^{2} \mathrm{HOD}$, Department of Anatomy, All India Institute of Medical Sciences, Bhubaneswar - 751 019, Odisha, India.

\section{Correspondence} \\ Divia Paul A. \\ Junior Research Fellow, Department of Anatomy, Yenepoya M edical College, Mangalore - 575 018, Karnataka, India. \\ Mobile : +917204215468 E-mail : divia_manoj@yahoo.com
}

\begin{abstract}
Variations in the extensor compartment of forearm are common and are significant to neurologists, surgeons and anatomists. The present case report describes a well-developed muscle belly medial to the tendons of extensor carpi radialis longus and extensor carpi radialis brevis. M uscle belly is originating from common extensor origin on lateral epicondyle and is inserted to base of third metacarpal; bilaterally. In addition to this, aberrant muscle fibres packed in a common connective tissue bundle over the dorsal digital expansion of left hand were also found. The above observations were noted during routine dissection of a 50 year old Indian male cadaver. The variant muscle having common extensor origin and muscle fibres over dorsal digital expansion were identified and protected. The blood supply and nerve supply were noted. The clinical significance of the variation is explained in detail.
\end{abstract}

Keywords: Extensor muscles of forearm, Dorsal digital expansion, Common extensor origin, Aberrant muscle fibres.

\section{Introduction}

Variations in muscles especially presence of additional bellies and presence of tendons of existing muscles in unusual locations might misguide surgical procedures. Such muscles may stimulate soft tissue tumors or can result in nerve compressions ${ }^{[1]}$. From an embryological perspective, the extensor muscles of the forearm extricates into three parts. The radial portion differentiates into the brachioradialis, extensor carpi radialis longus (ECRL) and extensor carpi radialis brevis (ECRB). Futher separation results in a superficial and deep portion. Extensor digitorum communis, extensor carpi ulnaris and extensor digiti minimi are seen in the superficial portion. The deep portion, which is innervated by the posterior interosseus nerve, gives rise to the abductor pollicis longus and the extensor pollicis brevis on the lateral side. The

\begin{tabular}{|c|}
\hline Access this article online \\
\hline Quick Response Code \\
\hline
\end{tabular}

extensor pollicis longus and extensor indicis on the medial side ${ }^{[2]}$. The variations such as presence of an additional tendon or fleshy belly of extensor carpi radialis longus (ECRL) are uncommon though there are reports on the same. When a supplymentary tendon prevails, it passes either through the second compartment of the extensor retinaculum ${ }^{[3]}$ or through a separate compartment ${ }^{[4]}$. In very subtle cases, the tendon of ECRL may split and get inserted into the fibrous flexor sheaths of the fingers. This can amend the biomechanics of wrist ${ }^{[5]}$. The familiarity of variations in ECRL and ECRB muscles helps for improvement of efficiency in various professions. Categorization of entrapment or compressive neuropathies can be made easy for surgeons by having acquaintance of the variations. For an orthopaedician ; for operating on the fractures on the lower end of the humerus such as supracodylar fractures and on the distal ends for forearm bones such as colles or smith's fractures and fractures of the wrist. Anaesthetists involved in pain executions therapeutics on the upper limb and physiotherapists carrying out electromyography for evaluating and documenting the electrical activities of the forearm muscles the awareness may be helpful for an enhanced proficiency. The clinical anatomy and morphology of the variant muscle discussed in this study. 


\section{Case Report}

During routine dissection for the first year medical students, Department of Anatomy, All India Institute of Medical Sciences, Bhubaneswar, Odisha the following variations were observed and noted. A fully developed additional muscle belly was found on the extensor compartment of the forearm, bilaterally on a approximately 50 year old male cadaver. In addition, fine granulated chips of muscle fibers packed in a separate connective tissue bundle were observed over the left side hand region over the dorsal digital expansion.

The additional muscle belly was found medial to extensor carpi radialis longus (ECRL) and extensor carpi radialis brevis (ECRB) tendons. The variant muscle was taking its origin from common extensor origin on the lateral epicondyle along with other extensor tendons. The additional muscle belly had a fleshy part and a tendinous part. The muscle was inserted to lateral side of the base of third metacarpal and was medial to the insertions of extensor pollicis longus (EPL), extensor carpi radialis longus (ECRL) and extensor carpi radialis brevis (ECRB) tendons. The morphometric analysis of the additional muscle belly was corresponding to the dimensions of the normal extensor tendons [Figure: 1,2]. The innervations and blood supply were confirmed to be the posterior interrosseous nerve and anterior interrosseous artery.

The fine fragments of muscle fibers were observed over the tendinous part of extensor digitorum, extensor pollicis longus and extensor carpi ulnaris tendons confirming their attachment to the respective tendons. Nerve supply and blood supply were posterior interrosseous nerve and anterior interrosseous artery. The muscle fibres seen over the extensor digitorum tendon were of $4 \mathrm{~cm}$ long. The leftovers were of $1 \mathrm{~cm}$ long and over extensor pollicis longus and extensor carpi ulnaris tendons respectively [Figure: 3].

\section{Discussion}

An anomalous muscle in the forearm extensor compartment is of academic interest. However, these muscles can create surgical complication when they
Fig. 1 : Right side muscle variant

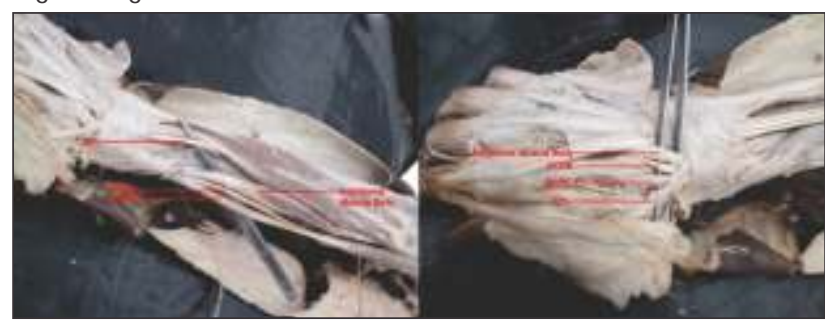

- ECRL-Extensor Carpi Radialis Longus

- ECRB-Extensor Carpi Radialis Brevis

- EPL-Extensor Pollicis Longus

Fig. 2 : Left side muscle variant with fine granules of aberrant muscle fibers over extensor retinaculum

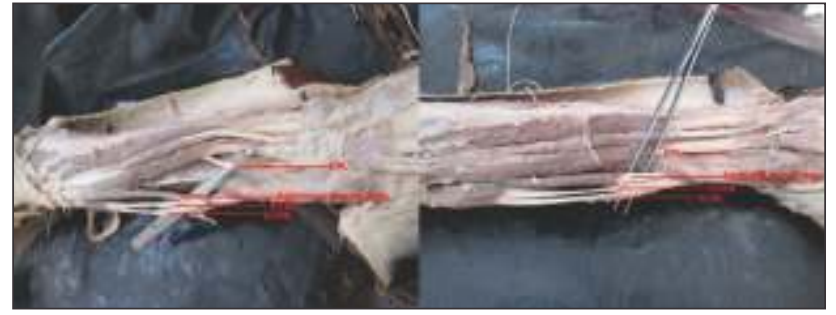

- ECRL-Extensor Carp i Radialis Longus

- ECRB-Extensor Carpi Radialis Brevis

- EPL-Extensor Pollicis Longus

Fig. 3 : Aberrant muscle fibres on the extensor digital expansion of forearm

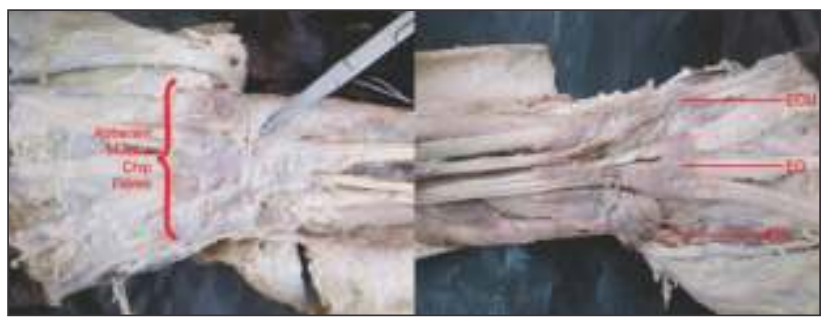

- ECU-Extensor Carpi Ulnaris

- ED-Extensor Digitorum

- EPL-Extensor Pollicis Longus

outturn in to clinical manifestations symptoms or spawn hardship to discriminate it from soft tissue tumors ${ }^{[6]}$.

M arked variations from the normal patterns are rarely seen in superficial group of extensors. Occasionally aberrant muscle slips are present among the superficial group of extensors of forearm ${ }^{[7]}$. An additional belly of extensor carpi radialis longus (ECRL) with a thin tendon ${ }^{[8]}$ were observed in four cases; in a study of hundred limbs for variations in the forearm extensor musculature.

The accessory slip originating from the ulnar side of the ECRL which gorge from the ulnar to radial side before 
insertion has also been reported ${ }^{[9]}$. Presence of an additional belly of ECRL on its ulnar side has also been reported by Chakravarthi ${ }^{[1]}$. The tendon of this crossed from the medial to lateral side superficial to the tendon of ECRL and was inserted to the second metacarpal bone. Thus among the reported variations, the presence of an additional belly on the ulnar side is more incessant compared to the one on the radial side. In the current case also there is an additional belly on the ulnar side of the ECRL.

The findings of the present study synchronizes with the above mentioned studies.

\section{In contrast to this,}

Muscular variation of the extensor compartment of the forearm is unusual and the variation in the superficial group of extensors is rarely observed ${ }^{[4]}$. Comparative anatomical studies have suggested that the superficial portion exhibits noticeable stability with the major divisions of the phylum of the animal species, while the deep portion turn-up to be extremely volatile and has withstood considerable evolutionary changes, which is observed by eloquent variation in its expression in different species of primates ${ }^{[2]}$.

The present study does not co-relate with these findings.

No comparative study was obtained for the aberrant muscle fibers packed in a common connective tissue

\section{References}

1. Chakravarthi KK. Unusual unilateral muscular variations of the flexor compartment of forearm and hand-A case report. Int J M ed health sci. 2012; 1(3):93-8.

2. Tan ST, Smith PJ. Anomalous extensor muscles of the hand: a review. J Hand Surg-Brit Eur. 1999; 24(3):449-55.

3. Sawant SP. The cadaveric study of extensor carpi radialis longus muscle on the developmental basis. Int J M ed Res Pharm Sci. 2013; 3(11):3842.

4. Claassen $\mathrm{H}$, Wree A. Multiple variations in the region of $\mathrm{Mm}$. extensores carpi radialis longus and brevis. Ann Anat. 2002; 184(5):489-91.

5. Jetti R, Nair V, Nair RV, M ookambika R, Somayaji K. Variant insertion of extensor carpi radialis longus in a South Indian cadaver. Int J Anat Var. 2010;3:86-7.

6. Nayak SR, Vadgaonkar R, Krishnamurthy A, Prabhu LV. An anomalous muscle in the forearm extensor compartment. Clin J. 2009; 64(3):262-3.

7. Hollinshead WH, Anatomy for Surgeons: The Back and Limbs. Harper and Row Publishers, New York. 1969; 3(2): pp.428-41.

8. Rao M, Vollala VR, Bhat SM, Bolla S, Samuel VP, Pamidi N. Four cases of bundle over the tendinous part of extensor muscles over dorsal aspect of hand unilaterally in the present study.

EMG studies and MRI Scan can confirm pre-operative diagnosis and to dodge superfluous intervention and complication thereof. Its presence can also be taken such as tendon transfers and reconstructive studies ${ }^{[10]}$.The tendon of ECRL is being used extensively in hand reconstructive surgeries ${ }^{[11,12,13]}$. Presence of additional bellies might prove to be a windfall to the patient. However a preoperative M RI scan of the forearm is obligatory to affirm the presence of such a variation. The additional tendon at the wrist might choke the spaces deep to the extensor retinaculum leading to the compression of the posterior interosseous nerve indirectly. This may consequence in deep-rooted wrist pain. The knowledge of occurrence of an additional belly may be of emphasis during injection of steroids (cortisol) in cases for tennis elbow or golfers elbow ${ }^{[14]}$.

\section{Conclusion}

Although the existence of variant muscle may be asymptomatic in most cases, it is necessary for surgeons to be attentive of variations of extensor muscles. This may be included in the differential diagnosis of a soft tissue mass on the dorsum of hand. The morphometric calibrations of the supplementary muscles and their tendons will help surgeons to execute tendon transfer in the ante brachial and carpal region in a safer context.

variations in the forearm extensor musculature in a study of hundred limbs and review of literature. Indian J Plast Surg. 2006; 39(2):141.

9. Cankur NS, Ozdemir ST. Accessory slip of the extensor carpi radialis longus muscle. Gazi M ed J. 2003; 14:197-99.

10. Sukumaran TT, M athew AJ. " Pozzi". Int] Anat Var. 2011; 4

11. Al-Qattan M. Two-staged extensor tendon reconstruction for zone 6 extensor tendon loss of the fingers: indications, technique and results. J Hand Surg Eur Vol. 2015; 40(3):276-80.

12. Chetta MD, Ono S, Chung KC. Partial extensor carpi radialis longus turn-over tendon transfer for reconstruction of the extensor pollicis longus tendon in the rheumatoid hand: case report. J Hand Surg Am. 2012; 37(6):1217-20.

13. Kerver AL1, Carati L, Eilers PH, Langezaal AC, Kleinrensink GJ, Walbeehm ET. An anatomical study of the ECRL and ECRB: feasibility of developing a preoperative test for evaluating the strength of the individual wrist extensors. J Plast Reconstr Aesthet Surg. 2013; 66(4):543-50

14. Edwards SG, Calandruccio JH. Autologous blood injections fo refractory lateral epicondylitis. J Hand Surg Am. 2003; 28(2):272-278. 